\title{
Alterations in self-perceptions following childhood onset of spinal cord injury
}

\author{
Colin Gorman ${ }^{2}$, Paul Kennedy ${ }^{1}$ and Lucy R Hamilton ${ }^{1}$ \\ ${ }^{1}$ National Spinal Injuries Centre, Stoke Mandeville Hospital NHS Trust, Aylesbury, Bucks HP21 8AL, UK; \\ ${ }^{2}$ Psychology Department, University of Ulster, N. Ireland
}

\begin{abstract}
Most research into psychological aspects of spinal cord injury (SCI) has focused on adult onset. This is a retrospective study of self-perceptions following a childhood onset of SCI. Self-esteem, depression and self-perception were examined in 86 people who had a traumatic SCI before the age of 16. Depression was measured using the Beck Depression Inventory, and self-esteem using the Culture-Free Self-Esteem Inventory Self-perception was measured using a scale developed for this study, consisting of 20 adjectives, with participants themselves on dimensions of 'As I am', 'As I would be without the injury', and 'as I would be ideally'. The participants' self perceptions of the injured self and the uninjured self were found to be significantly different on only nine of the 20 adjectives. Low self-perception was found to be associated with low self-esteem and high depression levels, but independent of age at injury, level of injury and gender. The change noted by the participants between how they see themselves now and how they would have been without the injury is not as great as might have been expected.
\end{abstract}

Keywords: childhood onset; spinal cord injury; psychology; self-perception

\section{Introduction}

Children under the age of 16 account for $4.5 \%$ of the spinal cord injured population. ${ }^{1}$ Medical problems associated with childhood spinal cord injuries are mainly those involving growth and possible scoliosis. Adolescence is a critical time for psychological and physical development, however, most previous research into the psychological aspects of spinal cord injury has focused on adult onset.

The adult literature suggests that, as well as the obvious physical changes in lifestyle that a spinal cord injured patient must undergo, there are many psychological aspects involved in adjustment and future management. Research into the psychological impact of spinal cord injuries notes depression, anxiety, and negative self-concept as being the most prevalent. There is some debate, not only about the prevalence of post-injury psychological disruption, but about the duration and long-term implications. Estimates of the prevalence of depression, anxiety, and self-esteem have decreased considerably in recent years. ${ }^{2,3}$

Depression was previously believed to be an essential part of the spinal cord injured patient's experiences. Stage theories have been proposed ${ }^{4-8}$ that included a period of depression for successful

Correspondence: P Kennedy adjustment to the injury. Other studies ${ }^{3}$ suggest that adjustment is possible without depression, and that only a minority of the SCI population experience depression. In a seminal review, Frank et $a l^{9}$ concluded that depression was not a universal phenomenon or a necessary precursor of adjustment to SCI. Recent estimates of the incidence of depression range from $25-34 \%{ }^{2,3}$ Estimates of the prevalence of anxiety in the SCI population range from as much as $25 \%{ }^{2}$ down to no significant difference from the general population $(5 \%){ }^{10,11}$

Negative self-concept has previously been associated with physical disability in both children ${ }^{12}$ and adults, ${ }^{13}$ and negative body image with suicidal tendency in depressed adolescents. ${ }^{14}$ In contrast to these findings, studies in SCI have shown no significant difference in the long term exists between SCI and non-SCI groups. ${ }^{15}$ Green et $a l^{16}$ even showed that those with SCI score above scale norms on some aspects of selfconcept such as personal, moral-ethical, and social self indices.

Research into long term adjustment has been able to identify some variables which may predict favourable psychological outcome. Woodrich and Patterson ${ }^{17}$ found that severity of injury did not appear to be a significant factor related to good overall adjustment, whereas gender did, with females showing better adjustment. In addition, younger patients were found to adjust better to SCI than 
older patients. Woodrich and Patterson ${ }^{17}$ suggested that young people may be more flexible and open to new experiences and directions than older people. Whereas, Frank et $a l^{18}$ confirmed that the experience of subjective life stress was not buffered by the age of the SCI patient, although the mean age of this sample was 30 , and 27 was the dividing point between young and old. More recent research has suggested that age is not predictive of good or bad adjustment. Kennedy et $a l^{3}$ matched people who were injured as children under 16 years with those who were injured as adults and found that there was no significant difference between the two groups on depression, self-esteem, or overall self-perception. In addition, those injured as children were not more accommodating to the injury than those injured later in life, both groups adjusting with equal success. Decker and Schultz ${ }^{19}$ found that many people of all ages are able to adjust to having a spinal cord injury and even take something positive out of the experience.

The aim of this study is to look more specifically at the self-perceptions of those from the spinal cord injured population who were injured as children, before the age of 16 . The study will compare how these particular individuals perceive themselves as they are now, as they think they would be without the injury, and as they would want to be ideally, using adjectives on a rating scale. In addition, this study will examine the relationship between selfperception and level of injury, age at injury, gender, self-esteem and depression. The specific hypotheses of this study are:

(1) There will be a significant difference between how people with a childhood SCI perceive themselves now and how they perceive they would be without the injury, as measured by the adjective ratings.

(2) Age at injury, level of injury and gender will not make any significant difference to self-perception.

(3) Levels of self-perception will differ significantly with depression and negative self-esteem

\section{Method}

\section{Subjects}

From a review of the files of the National Spinal Injuries Centre (NSIC), 645 patients were identified as having received their injury before their 16th birthday. Excluding injuries not of sudden onset removed 271 patients, and a further 123 were excluded because they had not been admitted to the NSIC until at least one year post-injury. Twentyfour were deceased and 73 were living abroad. The remaining 154 were each sent a letter inviting them to attend the outpatient department for a series of physical and psychological assessments. Sixteen were untraceable and 52 declined the invitation, resulting in 86 people ( $56 \%$ of the defined population). All of these participants met the criterion of at least 4 years since injury, used to ensure that no participants were still in the initial phase of disruption and adjustment, which is generally considered to last 2 or more years.

The ratio of male : female was $46: 40$, consisting of 54 paraplegics and 32 tetraplegics. The mean age at injury was 12.2 years and the mean time since injury, 19.9 years.

\section{Materials}

(1) The Self-Perception Scale, which was developed for this study and adapted from existing measures such as the acceptance of disability scale $\left(\right.$ Linkowskii ${ }^{20}$ ) and self-concept scale (Tyer$\left.\operatorname{man}^{21}\right)$. The self-perception scale consisted of 20 bipolar adjectives on a five-point Likert-type scale, eg popular, (a) represented very unpopular, (b) slightly unpopular, (c) neither popular or unpopular, (d) slightly popular, and (e) very popular. Higher scores reflected greater association with the adjective. The measure was completed three times on the dimensions of (a) 'As I am', (b) 'As I would be without the injury', and (c) 'As I would be ideally'. This provided three scores for each of the 20 items.

(2) The Beck Depression Inventory (BDI). ${ }^{22}$ This is a scale widely used in assessing depression and often used with SCI. It consists of 21 statements exploring affective and somatic aspects of depression. Whilst there is some indication that it may inflate estimates because of the somatic based items $^{23}$ it has been shown to be generally reliable. $^{24}$

(3) The Culture-Free Self-Esteem Inventory (SEI $)^{25}$ which gives scores for overall self-esteem, general self-esteem, social self-esteeem, and personal selfesteem, together with the measure of social desirability response bias.

\section{Procedure}

Ethical approval was obtained for the study from the local ethics committee. Each person in the population identified from the NSIC records was sent a letter requesting participation in the study, telling them that the purpose of the study was to improve the treatment of children with spinal cord injury. A follow-up letter was sent to non-responders and, if necessary, a telephone call was also made. The 86 who consented were interviewed individually. At the beginning of the interview the purpose of the study was again explained, the participants were reassured about the confidentiality of the information, and required to sign a consent form. The questionnaires were verbally administered to some tetraplegics who were unable to fill them in. Travelling expenses were paid to those who requested them, but there was no other incentive to participate. 

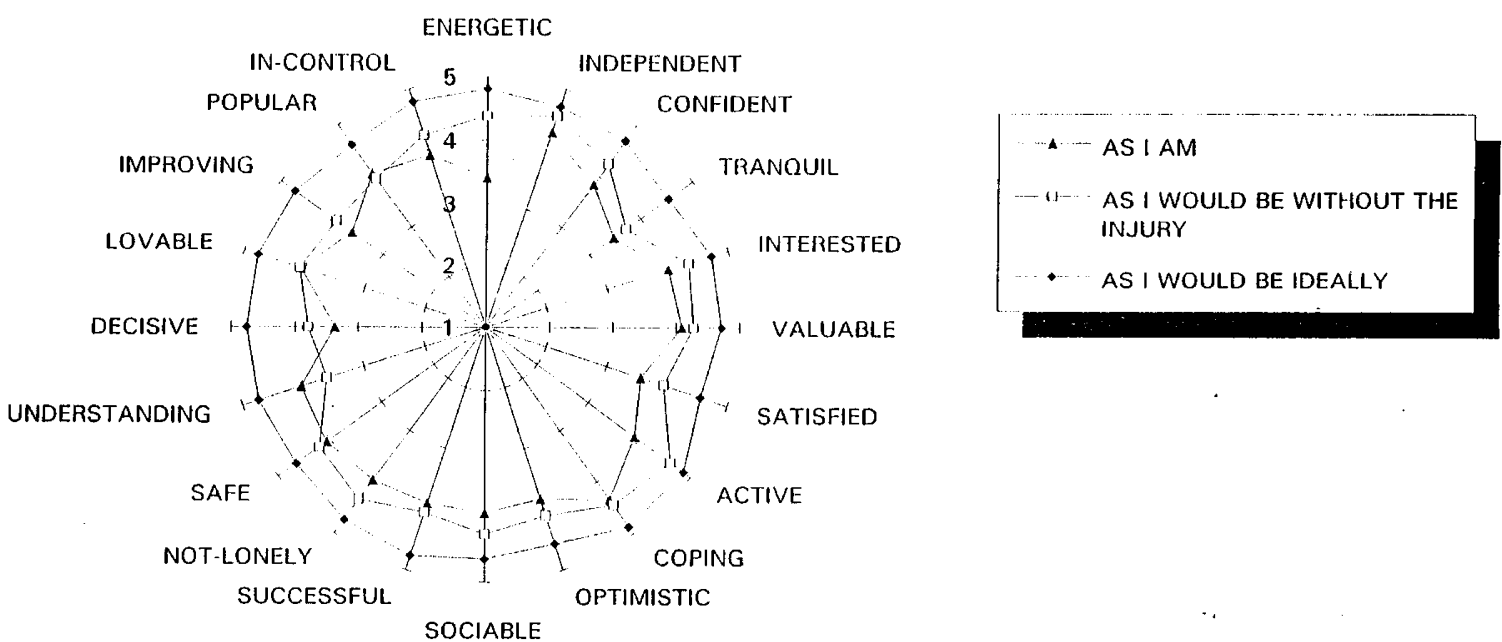

Figure 1 A comparison of mean scores of perceptions of 'as I am', 'as I would be without the injury' and 'as I would be ideally'

\section{Results}

Self-perception scale

The mean scores of the 20 adjectives on the selfperception scale are presented in Figure 1, relating to the three dimensions 'As I am', 'As I would be without the injury' and 'As I would be ideally'. There is an overall tendency for the uninjured self adjective score, as opposed to the injured self adjective score, to be closer in value to the ideal self adjective score. On only two adjectives, popular and understanding, do the 'As I am' scores exceed the 'As I would be without the injury' scores.

The differences between the mean ratings for each adjective on 'As I am', 'As I would be without the injury', and 'As I would be ideally' scales are compared in Table 1 using the $t$-test.

Levels of significance were corrected to account for the number of $t$-tests carried out, resulting in nine significant differences between how the participants perceive the self and the uninjured self. Of these nine differences, the participants only describe themselves to be more understanding as a result of the injury. The differences between the participants' ratings of 'As I am' and 'As I would be ideally' are significant for all adjectives. However, in comparing 'As I would be without the injury' with 'As I would be ideally', the only non-significant difference is that for independent.

Age at injury, level of injury and gender

T-tests were used to compare the lower and upper quartiles of age at injury $(<11$ years and $>15$ years, respectively) with each of the 20 adjectives. There were no signficant differences in 'As I would be without the injury' and only not-lonely was significant in the 'As I would be ideally' dimension $(P=0.046)$. Comparing the upper and lower quartiles of level of injury gave no
Table 1 t-probabilities of differences between ratings of: 'As I am' (A), 'As I would be without the injury' (B), and 'As I would be ideally' (C)

\begin{tabular}{llll}
\hline Adjective & $P(\boldsymbol{A}-\boldsymbol{B})$ & $P(\boldsymbol{A}-\boldsymbol{C})$ & $P(\boldsymbol{B}-\boldsymbol{C})$ \\
\hline Energetic & $* * *$ & $* * *$ & $* * *$ \\
Independent & $\mathrm{NS}$ & $* * *$ & $\mathrm{NS}$ \\
Confident & $* *$ & $* * *$ & $* * *$ \\
Tranquil & $\mathrm{NS}$ & $* * *$ & $* * *$ \\
Interested & $* *$ & $* * *$ & $* *$ \\
Valuable & $\mathrm{NS}$ & $* * *$ & $* * *$ \\
Satisfied & $*$ & $* * *$ & $* * *$ \\
Active & $* * *$ & $* * *$ & $* *$ \\
Coping & $\mathrm{NS}$ & $* * *$ & $* * *$ \\
Optimistic & $*$ & $* * *$ & $* * *$ \\
Sociable & $* *$ & $* * *$ & $* *$ \\
Successful & $\mathrm{NS}$ & $* * *$ & $* * *$ \\
Not-Lonely & $* *$ & $* * *$ & $* * *$ \\
Safe & $\mathrm{NS}$ & $* * *$ & $* * *$ \\
Understanding & $* *$ & $* * *$ & $* * *$ \\
Decisive & $* *$ & $* * *$ & $* * *$ \\
Lovable & $\mathrm{NS}$ & $* * *$ & $* * *$ \\
Improving & $* *$ & $* * *$ & $* * *$ \\
Popular & $\mathrm{NS}$ & $* * *$ & $* * *$ \\
In-Control & $\mathrm{NS}$ & $* * *$ & $* * *$ \\
\hline
\end{tabular}

$*=P<0.05 ; * *=P<0.01 ; * * *=P<0.001$

significant results on either 'As I would be without the injury' or 'As I would be ideally'. The responses of males and females were compared and significant differences were only seen for coping and safe $(P<0.05)$ in ratings of 'As I would be ideally'.

\section{Depression}

The lower quartile $(\mathrm{BDI}<3)$ and the upper quartile $(\mathrm{BDI}>10)$ for depression were compared using $t$-tests. On the 'As I am' dimension significant differences were 
found for all but three of the adjectives (independent, valuable and understanding). Four adjectives, confident, active, safe, and improving, were significant only at the 5\% level. Adjusting the level of significance for the large number of $t$-tests carried out left these differences also non-significant. The participants from the lower quartile of depression gave an overall mean rating of 4.24 to the adjectives, whereas those in the upper quartile had a mean rating of 3.37 . For ratings of 'As I would be without the injury' there is a general tendency for the participants with a higher level of depression to have lower expectations of how they would have been. The overall rating given by the lower quartile, the less depressed group, to the adjectives was 4.33 and 4.10 for the upper quartile, though no single adjective showed a significant difference. For ratings of 'As I would be ideally' those participants with a higher depression score rate themselves lower than the less depressed participants on the majority of the adjectives. Three of these differences are significant at the $1 \%$ level (satisfied, successful, and popular). Those who scored higher on depression rated coping significantly higher $P<0.01)$ than those with lower depression scores.

\section{Self-esteem}

The lower quartile of self-esteem measured by the SEI was $\mathrm{SEI}<22$, and the upper quartile was $\mathrm{SEI}>29$. The differences between the high self-esteem and low-esteem groups on each of the dimensions were measured using $t$-tests. In relation to how the participants perceived 'As I am' there were significant differences between high and low self-esteem groups on all but four adjectives (independent, understanding, improving, and active) having adjusted the significance level to 0.01 for the number of tests involved. The group scoring low in self-esteem had an overall mean rating of 3.3 for the adjectives in this dimension and the higher self-esteem participants had a mean rating of 4.4. Although the low and high self-esteem groups did not differ significantly in ratings of 'As I would be without the injury', high self-esteem participants had a tendency to be associated with a more positive view of 'As I would be ideally', none of the differences being significant once the level of significance was corrected.

Further analysis using the Pearson product moment coefficient showed that depression was highly correlated with self-esteem $(r=-0.74, P<0.001)$, although no correlations were found with age at injury, level of injury or gender.

\section{Discussion}

Before looking in further detail at the results, it is important to note the sampling bias in this study. Of the 154 people contacted about the study $44 \%$ did not participate, $76 \%$ of whom actually declined the invitation. It is a possibility that the psychological profile of this group differed significantly from that of the participant group, a group with poor psychological adjustment being less likely to respond and take part in research.

The first hypothesis of this study, that there would be a significant difference between the adjective ratings given by people with a childhood SCI to describe themselves now and without the injury, is not fully supported by the results. A significant difference was found on only nine out of the 20 adjectives between self and uninjured-self ratings. This could be due to the sampling bias of the participant group. The fact that the participants perceived themselves to be more popular and understanding with the injury is encouraging and suggests that, as in Decker and Schultz's ${ }^{19}$ findings, they obtain something positive from the experience. It is, however, difficult to generalize to the SCI population because of the sampling bias.

In this study age at injury, level of injury, and gender do not generally make a significant difference to selfperception adjective ratings, as hypothesized. Both Kennedy et $a l^{3}$ and Frank et $a l^{18}$ found that age at injury did not make a difference to overall adjustment, wheras Woodrich and Patterson ${ }^{16}$ suggest that age is an important factor. The results here lend support to the findings that there is no effect of age at injury, measured in this instance within a childhood injury group. Level of injury does not make a difference to self-perception, in agreement with Woodrich and Patterson's findings. ${ }^{17}$ However, Woodrich and Patterson ${ }^{17}$ found females better able to adjust to their injury, whereas this study suggests that, in terms of self-perception, there is no significant difference between males and females. These results could again be confounded by the sampling bias present in the study.

Self-perception did differ with levels of depression and negative self-esteem. Low self-esteem and high depression scores were associated with a significantly more negative perception of the self and to a lesser extent, the ideal self. This suggests that as well as lowering self-opinion, low self-esteem and high depression can lower aspirations in spinal cord injured people. Orback et al ${ }^{14}$ reported a link between depression and negative body image in adolescents, and here we have found a link between depression and self-perception in a group of SCI people with childhood injuries. Furthermore, there is a high negative correlation between levels of depression and self-esteem.

In addition to the sampling bias of the group invited for participation, generalizations to the SCI population are limited by the people who were excluded from invitation due to death or living abroad. Possibly these people also had a different psychological profile. This study does, however, give an important insight into how a group of SCI people who were injured as children perceive themselves in the long term. A study by De Loach and Greer ${ }^{26}$ suggests that after a spinal cord injury, in order to achieve stability and maintain the same quality of life 
satisfaction, a person redefines situations and adapts new value systems. The present study shows that values such as independence and control, success and value, popularity and being loved, ability to cope, and safety are not values that needed to be redefined in the SCI person, and that personal ideals are not noticeably different from the general population.

In conclusion, it appears that self-perception after a childhood spinal cord injury is associated with psychological factors rather than organic variables such as gender, age at injury, and level of injury. We cannot determine from this study whether low selfesteem and high depression levels are a cause or an effect of low self-perception. The childhood injured group studied here do note some change between how they see themselves and how they would have been, but this change is not as great as one might have expected. Intervention for the management of mood disturbances may help to decrease the perceived severity of the injury and hence the perceived severity of any loss resulting from the injury.

\section{Acknowledgements}

The support of the National Spinal Injuries Centre, Stoke Mandeville, and the people who cooperated with the study are greatly appreciated.

\section{References}

1 Stover SL, DeLisa JA, Whiteneck GG Spinal Cord Injury. Clinical Outcomes from the Model Systems. Aspen: Gaithersburg 1995, pp $21-55$.

2 Hancock K et al. Anxiety and depression in the first year of spinal cord injury: a longitudinal study. Paraplegia 1993; 31: 349 - 357.

3 Kennedy P, Gorsuch N, Marsh N. Childhood onset of spinal cord injury: self-esteem and self-perception. British Journal of Clinical Psychology 1995; 34: 581 - 588.

4 Peter A. Psychological aspects of spinal cord injury. Maryland State Medical Journal 1975; 24: 65-69.

5 Guttmann L. Spinal Cord Injuries: Comprehensive Management and Research. 2nd edn. Blackwell Scientific Publications: Oxford 1976.

6 Stewart T. Coping behaviour and the moratorium following spinal cord injury. Paraplegia 1977; 15: 338 - 342.

7 Bracken M, Shepherd M. Coping and adaption to acute spinal cord injury: A theoretical analysis. Paraplegia 1980; 18: 74-85.
8 Tucker S. The psychology of spinal cord injury: patient/staff interaction. Rehabilitation Literature 1980; 41: 114-121.

9 Frank RG, Elliott TR, Corcoran JR, Wonderlich S. Depression after spinal cord injury: is it necessary? Clinical Psychology Review 1987; 7: 611-630.

10 Schultz R, Decker S. Long term adjustment for physical disability: The role of social support, perceived control and self-blame. Journal of Personality and Social Psychology 1985; 48: $1162-1172$.

11 Cook D. Psychological adjustment to spinal cord injury: incidence of denial, depression and anxiety. Rehabilitation Psychology 1979; 26: $97-104$.

12 Harvey D, Greenway A. The self-concept of physically handicapped children and their non-handicapped siblings: an empirical investigation. Journal of Child Psychology and Psychiatry 1984; 25: 273 - 284.

13 Wright B. Physical Disability: Psychological Approach. 2nd edn. Harper and Row: New York 1983.

14 Orback I, Lotem-Peleg M, Kedem P. Attitudes towards the body in suicidal, depressed and normal adolescents. Suicide and Life Threatening Behaviour 1995; 25: $211-221$.

15 Mayer J, Eisenberg M. Self-concept and the spinal cord injured: an investigation using the Tennessee self-concept scale. Journal of Consulting and Clinical Psychology 1982; 50: 604-605.

16 Green B, Pratt C, Grisby T. Self-concept among persons with long term spinal cord injury. Archives of Physical Medicine and Rehabilitation 1984; 65: 751-754.

17 Woodrich F, Patterson J. Variables related to acceptance of disability in persons with spinal cord injuries. Journal of Rehabilitation 1983; 49: 26-30.

18 Frank RG, Elliott TR, Buckelew S, Hart A. Age as a factor in response to spinal cord injury. American Journal of Physical Medicine and Rehabilitation 1988; 67: $128-131$.

19 Decker SD, Schultz R. Correlates of life satisfaction and depression in middle-aged and elderly spinal cord injured persons. The American Journal of Occupational Therapy 1985; 39: $740-745$.

20 Linkowski DC. A scale to measure disability. Rehabilitation Counselling Bulletin 1971; 4: 236-244.

21 Tyerman A. Self-concept and Psychological Change in the Rehabilitation of the Severely Head Injured Person. Unpublished Phd Thesis: University of London, 1987.

22 Beck AT, Rush AJ, Shaw BF, Emery G. Cognitive Therapy of Depression. Guildford Press: New York, 1979.

23 Jacob K, Zachariah K, Bhattacharji S. Depression in spinal cord injury: methodological issues. Paraplegia 1995; 33: $377-380$.

24 Judd F, Brown D, Burrows G. Depression, disease and disability: applications to patients with traumatic spinal cord injury. Paraplegia 1991; 29: 91 - 96.

25 Battle J. Culture-free Self-esteem Inventory. Special child publications: Seattle, 1981.

26 De Loach C, Greer B. Adjustment to Severe Physical Disability. A Metamorphosis. McGraw-Hill: New York, 1981. 